\title{
Eficiencia del uso del agua de un viñedo \\ y su relación con porosidad del suelo
}

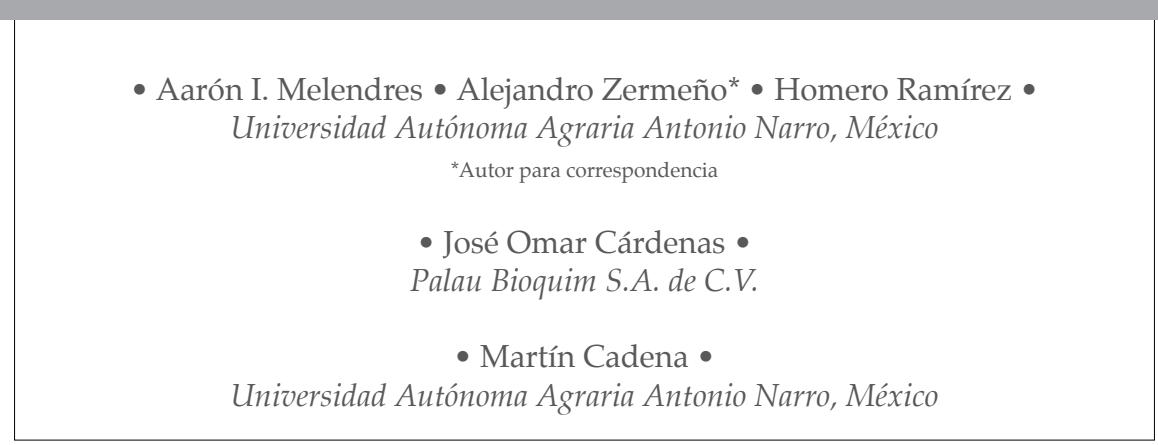

DOI: $10.24850 /$ j-tyca-2017-05-04

Resumen

Melendres, A. I., Zermeño, A., Ramírez, H., Cárdenas, J. O., \& Cadena, M. (septiembre-octubre, 2017). Eficiencia del uso del agua de un viñedo y su relación con porosidad del suelo. Tecnología y Ciencias del Agua, 8(5), 57-69.

El objetivo del estudio fue determinar el efecto de la capacidad de retención de humedad del suelo debido a la porosidad $(\eta)$ y su relación con la eficiencia intrínseca del uso del agua $\left(E F_{i}\right)$ y la productividad marginal del agua $(P M A)$ de una plantación de vid (Vitis vinifera L.) cv Shiraz. El estudio se realizó en dos viñedos de diferente porosidad en la Vinícola San Lorenzo, Parras, Coahuila, en el ciclo de producción marzo-septiembre de 2014. La $\eta$ se determinó por medio de la densidad aparente y de partículas. Los cambios en la humedad del suelo se midieron con sondas TDR en cada plantación y la $E F_{i}$ con determinaciones de asimilación de $\mathrm{CO}_{2}$ y tasa de evapotranspiración colocando un sistema Eddy en cada plantación. La porosidad de una de las plantaciones fue $0.385 \mathrm{~cm}^{3} / \mathrm{cm}^{3}$ (mayor porosidad) y la otra $0.306 \mathrm{~cm}^{3} / \mathrm{cm}^{3}$, que correspondió a una diferencia de $25.82 \%$. La diferencia en la porosidad del suelo significó una notable diferencia en el contenido volumétrico de agua del suelo, que resultó en una mayor tasa de evapotranspiración y de asimilación de bióxido de carbono por el cultivo. La mayor disponibilidad de agua en el suelo no tuvo efecto en la eficiencia intrínseca del uso del agua, sin embargo, la productividad marginal del agua fue, en promedio, $24.64 \%$ mayor en el viñedo de mayor porosidad a través de las etapas de desarrollo de las plantas.

Palabras clave: covarianza Eddy, evapotranspiración, fotosíntesis.
Melendres, A. I., Zermeño, A., Ramírez, H., Cárdenas, J. O., \& Cadena, M. (September-October, 2017). Water use efficiency in a vineyard and its relationship with soil porosity. Water Technology and Sciences (in Spanish), 8(5), 57-69.

The objective of this study was to determine the effect of the waterholding capacity of soil given its porosity ( $\eta)$, and its relationship with the intrinsic water-use efficiency (WUEi) and the marginal productivity of the water (MPW), in a vineyard planted with Vitis vinifera L. (Shiraz variety). This study was conducted in two vineyards having different soil porosity, on land belonging to the San Lorenzo wine company in Parras, Coahuila during the March-September growing cycle, 2014. The $\eta$ was calculated based on the bulk and particle density. Changes in the soil's water content were evaluated at each vineyard using TDR probes, and the WUEi was determined based on $\mathrm{CO}_{2}$ assimilation and evapotranspiration rates, with an Eddy system in each vineyard. The soil porosity reached $0.385 \mathrm{~cm}^{3} / \mathrm{cm}^{3}$ (highest value) in one of the vineyards and $0.306 \mathrm{~cm}^{3} / \mathrm{cm}^{3}$ in the other, which corresponded to a difference of $25.82 \%$. This difference in soil porosity indicates a large difference in the volumetric soil water content, resulting in higher evapotranspiration and $\mathrm{CO}_{2}$ assimilation rates. The highest water availability did not affect the intrinsic water-use efficiency. And throughout the growing stages of the plants, the marginal productivity of the water was on average $26.64 \%$ higher in the vineyard that had more soil porosity.

Keywords: Eddy Covariance, evapotranspiration, photosynthesis.

Recibido: $12 / 11 / 2015$

Aceptado: 28/03/2017

ISSN 0187-8336 • Tecnología y Ciencias del Agua, vol. VIII, núm. 5, septiembre-octubre de 2017, pp. 57-69 


\section{Introducción}

El contenido de agua en el suelo determina el grado de crecimiento de las plantas y el rendimiento de los cultivos. Una óptima programación de la irrigación requiere mediciones continuas del agua en el suelo (Poltoradnev, Ingwersen, \& Streck, 2014). La distribución y proporción de los agregados del suelo inciden en las características físicas y químicas del suelo, porosidad y crecimiento del sistema radicular (Lipiec et al., 2007). Los cambios en la humedad de perfil superior del suelo dependen de la tasa de evapotranspiración de las plantas (función de las condiciones climáticas) y de las propiedades hidráulicas del suelo (Martínez-García, Pachepsky, \& Vereecken, 2014).

La porosidad del suelo es un factor importante en la infiltración, conductividad hidráulica y retención de agua. Bruschi-Gonçalves et al. (2010), y Hongshuo, Rogers y Munroe, (2015) describen la densidad aparente del suelo como unas de las principales propiedades que influyen en las variaciones espaciales de la humedad del suelo. Holland \& Biswas (2015) reportaron que las propiedades físicas del suelo que tienen un mayor efecto en la retención de agua del suelo en una plantación de vid fueron el contenido de arcilla y la densidad aparente del suelo.

El intercambio de bióxido de carbono y vapor de agua entre las hojas de las plantas y la atmósfera es regulado por los estomas; la relación de estos flujos determina la eficiencia intrínseca del uso del agua $\left(E F_{i}\right)$ y la productividad de las plantas (Lawson \& Blatt, 2014).

El balance de flujos de vapor de agua y bióxido de carbono depende de las repuestas de los estomas a los cambios del ambiente y la sincronía de éstos con el mesófilo para la demanda de $\mathrm{CO}_{2}$ (Lawson \& Blatt, 2014; McAdam \& Brodribb, 2014; Wang, Hills, \& Blatt, 2014; Xu, Li, Shao, Xu, \& Nugroho, 2014; Li et al., 2015).

La $E F_{i}$ (definida como la cantidad de carbono fijado en la fotosíntesis por unidad de agua transpirada) (Ago et al., 2014; Lawson \& Blatt, 2014) es usada como un indicador de la habilidad de las plantas para adaptarse al déficit hídrico en regiones semiáridas (Song, Zhu, Yan, Li, \& Yu, 2015). Sun, Yan, Kang y Ma (2013) mencionan que la eficiencia intrínseca del uso del agua se puede incrementar con el cierre estomático.

Li et al. (2015) evaluaron la $E F_{i}$ de un cultivo de vid cv Merlot Noir bajo diferentes condiciones ambientales de una región de clima seco. Mientras que en un cultivo de manzana (Malus. domestica) (cv Pacific Rose), Liu, Cheng, Ma, Zou y Liang (2012) evaluaron la relación entre la $E F_{i}$ bajo diferentes programaciones de riego y déficit hídrico. También se han realizado mediciones de la $E F_{i}$ en sabanas, bosques de clima templado, subtropical y de coníferas (Mahrt \& Vickers, 2002; Scanlon \& Albertson, 2004; Yu et al., 2007).

México tenía reportadas en 2014 más 29000 hectáreas de cultivo de vid (SIAP, 2015), con una producción de vinos de un poco más de $39 \mathrm{mi}$ llones de litros, teniendo un consumo per cápita de 0.53 litros (OIV, 2015). La industria mexicana la componen los estados de Baja California, como principal productor, Coahuila, Querétaro, Zacatecas, Guanajuato y Aguascalientes. En su conjunto ofrecen más de 350 etiquetas de vino (El Economista, 2013).

Dado que la porosidad del suelo influye en la capacidad de retención de agua y esto, a su vez, en la tasa de traspiración y fotosíntesis, el objetivo del presente estudio fue evaluar el efecto de la disponibilidad de agua en el suelo (por diferencias de porosidad) en la eficiencia intrínseca del uso del agua y su productividad marginal de un viñedo cv. Shiraz.

\section{Materiales y métodos}

\section{Descripción del sitio de estudio}

El estudio se realizó durante el ciclo de producción abril-septiembre de 2014, en una plantación de vid cv Shiraz de siete años de edad, ubicada en la Vinícola San Lorenzo, Parras de la Fuente, Coahuila, México, a $25^{\circ} 30^{\prime} \mathrm{N}, 102^{\circ} 11^{\prime} \mathrm{O}$, y 1505 de altitud; el clima es seco semiárido, con temperaturas promedio de 14 a $18{ }^{\circ} \mathrm{C}$, 
precipitación promedio anual de $366 \mathrm{~mm}$; los vientos dominantes son del este durante el ciclo de producción (INIFAP, 2015).

Para el estudio se delimitaron dos superficies de 5.2 ha (204 m en la dirección E-O, por $256 \mathrm{~m}$ dirección N-S) de diferente textura de suelo y distinta porosidad. Las plantas están alineadas en la dirección norte-sur, a $1.5 \mathrm{~m}$ entre plantas y $2.5 \mathrm{~m}$ entre hileras, en una densidad de 2620 plantas/ha. El riego en ambas secciones del viñedo se aplica por goteo diariamente $(2 \mathrm{~h})$, que para el gasto del emisor (2.1 LPH) y el ancho de mojado $(40 \mathrm{~cm})$ correspondió a una lámina de $7 \mathrm{~mm}$. La altura máxima de plantas (etapa de floración) fue de $2 \mathrm{~m}$. Cada superficie recibió el mismo manejo agronómico (riego, poda, fertilización y control fitosanitario) de acuerdo con las normas establecidas por la Vinícola San Lorenzo.

\section{Características físicas y químicas del suelo del área de estudio}

Las características físicas y químicas del suelo se obtuvieron del promedio de cinco muestras de la parte central de cada superficie. Las propiedades químicas determinadas fueron $\mathrm{pH}$, $\mathrm{CE}$, contenido de nitrógeno, fósforo, potasio y carbonatos totales. Las propiedades físicas fueron contenido de materia orgánica, arcilla, limo, arena, densidad aparente del suelo y densidad de partículas. La densidad aparente se determinó por el método del cilindro, utilizando una barrena de corazones, mientras que la densidad de partículas se obtuvo con el método del picnómetro. La porosidad del suelo $(\eta)$ se estableció con los valores de densidad aparente $(\rho b)$ y densidad de partículas $(\rho s)$, con la siguiente relación:

$$
\eta=1-\frac{\rho b}{\rho s}
$$

\section{Mediciones e instrumentación}

Las variaciones del contenido de agua en el suelo en cada sección (mayor y menor porosidad) del viñedo se determinaron colocando dos sondas TDR: una en el perfil de 0 a $30 \mathrm{~cm}$ y la otra de 30 a $60 \mathrm{~cm}$ de profundidad del suelo en cada sección del viñedo. Las sondas de cada viñedo se conectaron a un datalogger CR1000 (Campbell Sci. Inc., Logan, Utah, EE.UU), para un registro continuo de datos cada 30 minutos a través del ciclo de producción. La diferencia estadística en contenido de humedad en las dos secciones se evaluó con la prueba no paramétrica de Wilcoxon $(\alpha \leq 0.05)$.

Para determinar la eficiencia intrínseca del uso del agua de cada viñedo (mayor y menor porosidad), se obtuvo el flujo de calor sensible $(H)$, calor latente $(L E)$ y flujo de bióxido de carbono $\left(\mathrm{FCO}_{2}\right)$ entre el viñedo y la atmósfera, instalando un sistema Eddy (con los sensores correspondientes) en la parte media del extremo oeste de cada viñedo. Las ecuaciones correspondientes de los flujos mencionados (Ham \& Heilman, 2003) son:

$$
\begin{gathered}
H=\rho a^{*} C p^{*}\left(\overline{w^{\prime * T s^{\prime}}}\right)-0.51 * T a^{*} \frac{\rho a^{*} C p * L E}{L} \\
L E=L^{*}\left(\overline{\mathrm{w}^{\prime *} \rho_{w v v}^{\prime}}\right) \\
\mathrm{F}_{\mathrm{CO}_{2}}=\overline{w^{\prime *} \rho_{\mathrm{CO}_{2}}^{\prime}}
\end{gathered}
$$

Donde las unidades de $H$ y $L E$ son $\mathrm{W} \mathrm{m}^{-2}$ y las de $\mathrm{F}_{\mathrm{CO}_{2}}$ son $\mu$ mol $\mathrm{CO}_{2} \mathrm{~m}^{-2} \mathrm{~s}^{-1} ; \rho a, \mathrm{Cp}$ y $\mathrm{Ta}$ son la densidad, capacidad calorífica y temperatura del aire, respectivamente $\left(\mathrm{kg} \mathrm{m}^{-3}, \mathrm{~J} \mathrm{~kg}^{-1}\right.$ $\left.\mathrm{K}^{-1},{ }^{\circ} \mathrm{C}\right) ; \mathrm{Ts}$, la temperatura sónica $\left({ }^{\circ} \mathrm{C}\right) ; \rho_{w v^{\prime}}$ la densidad del vapor de agua en el aire $\left(\mathrm{kg} \mathrm{m}^{-3}\right)$; $w$, la velocidad vertical del viento $(\mathrm{m} / \mathrm{s}) ; L$, el calor de vaporización del agua $\left(\mathrm{J} \mathrm{kg}^{-1}\right), y \rho_{\mathrm{CO}_{2}}$ es la densidad de bióxido de carbono ( $\mu \mathrm{mol} \mathrm{m}^{-3}$ ). Las variables con símbolo de prima significan desviaciones respecto a la media y la barra horizontal sobre dos variables denota la covarianza entre las mismas para un determinado segmento de tiempo (30 min).

La velocidad vertical del viento y la temperatura sónica se midieron con un anemómetro sónico tridimensional (CSI-CSAT3, Campbell, 
Scientific, Inc., Logan, Utah, EUA); para obtener $\rho_{w v}$ y $\rho_{\mathrm{CO}_{2}}$ se usó un analizador infrarrojo de bióxido de carbono y vapor de agua de sendero abierto (Open Path $\mathrm{CO}_{2} / \mathrm{H}_{2} \mathrm{O}$ analyzer, LI-7500. LI-COR, Lincon, Nebraska, EUA). La temperatura del aire $(\mathrm{Ta})$ se midió a la misma altura que $T s$, con un sensor de temperatura y humedad relativa (HP45C, Vaisala, Inc., Woburn, MA, EUA). Los sensores mencionados se montaron en un poste a $3 \mathrm{~m}$ de altura sobre la superficie del suelo ( $2 \mathrm{~m}$ sobre el dosel de las plantas) en el extremo oeste y al punto medio de las hileras de plantas de cada viñedo.

El anemómetro sónico tridimensional se orientó hacia el este, para que el viento tuviera por lo menos $200 \mathrm{~m}$ de contacto con la superficie vegetal en la dirección este-oeste y $125 \mathrm{~m}$ en la dirección norte-sur, antes del contacto con los sensores. Los vientos provenientes del oeste (que impactaban en la parte posterior del 3-D Sonic anemómetro) no se consideraron en las determinaciones de los flujos. La frecuencia de operación de los sensores fue $10 \mathrm{~Hz}$ y los flujos se obtuvieron en promedio de $30 \mathrm{~min}$; los datos se almacenaron en el módulo de memoria de un datalogger CR1000 (Campbell, Scientific, Inc., Logan, Utah, EUA).

Para evaluar la precisión de las mediciones de los flujos se determinó el balance de energía sobre la superficie vegetal (Kim \& Verma, 1990; Zermeño-González, 2007; Pardo, Sánchez, Pérez, \& García, 2015) con la siguiente relación:

$$
R n=H+L E+G
$$

Donde $R n$ es la radiación neta; $H$ y $L E$ ya fueron definidos, y $G$ es el flujo de calor en la superficie del suelo. Las unidades de todas las variables son $\mathrm{W} \mathrm{m}^{-2}$. La $R n$ se midió con un radiómetro neto (modelo LITE, Keep and Zonen, Inc., Delft, Holanda), colocado a $1 \mathrm{~m}$ sobre el dosel de cada viñedo. El flujo de calor en la superficie del suelo $(G)$ se obtuvo midiendo el flujo de calor a $0.08 \mathrm{~m}$ bajo la superficie del suelo al punto medio de una hilera de plantas y al centro de un pasillo de cada viñedo, con un transductor de calor (modelo HFT3, Campbell
Scientific, Inc., Logan, Utah, EUA), y sumando los cambios de temperatura del suelo sobre cada transductor (Kustas, Prueger, Hatfield, Ramalingam, \& Hipps, 2000). El flujo de calor sensible $(H)$ y latente $(L E)$ se corrigieron por efecto de diferencia de densidad entre las masas de aire ascendentes y descendentes (Webb, Pearman, \& Leuning, 1980). El flujo de $\mathrm{CO}_{2}$ se corrigió con el mismo factor (Ham \& Heilman, 2003).

El intercambio neto de bióxido de carbono (NEE) entre el dosel de cada viñedo y la atmósfera $\left(\mu \mathrm{mol} \mathrm{CO} \mathrm{Cm}^{-2} \mathrm{~s}^{-1}\right)$ se obtuvo con la siguiente relación (Martens et al., 2004):

$$
N E E=\mathrm{F}_{\mathrm{CO}_{2}}+\frac{\Delta \rho_{\mathrm{CO}_{2}}}{\Delta t} * \mathrm{Z}
$$

Donde $\mathrm{F}_{\mathrm{CO}_{2}}$ es el flujo de bióxido de carbono medido con el método de la covarianza Eddy (ecuación (4)) (negativo hacia la superficie vegetal); $\Delta \rho_{\mathrm{CO}_{2}{ }^{\prime}}$ el cambio en la densidad de $\mathrm{CO}_{2}$ medido a la altura $Z$; $\Delta t$, el intervalo de tiempo (30 min); $Z$ es la altura sobre la superficie del suelo a la que se realizan las mediciones de flujo $(3 \mathrm{~m})$.

La eficiencia intrínseca del uso del agua de cada plantación fue la relación entre la asimilación neta de bióxido de carbono (NEE) y la tasa de evapotranspiración (Tasa_ET):

$$
E F i=\frac{N E E}{\text { Tasa_ET }}
$$

La tasa de evapotranspiración se obtuvo dividiendo el flujo de calor latente ( $L E$ ) por el calor de vaporización del agua $(L)$. Los cambios en la humedad del suelo a través del ciclo de producción de cada viñedo se determinaron colocando sondas TDR (modelo CS616; Campbell, Scientific, Inc., Logan, Utah, EE.UU). Las mediciones se realizaron en los estratos de 0 a 30 y 30 a $60 \mathrm{~cm}$ de profundidad, al punto medio entre dos plantas, al centro de una de las hileras de cada viñedo (de diferente porosidad). Los datos se registraron continuamente a una frecuencia de media hora. Los registros de las sondas se calibraron con mediciones directas de humedad del suelo. 
Para comparar la productividad del agua de los viñedos en las diferentes etapas de desarrollo, se determinó la productividad marginal del agua del ecosistema, que se definió como el incremento en la tasa de asimilación de bióxido de carbono por cada mol adicional de agua evapotranspirada a una preestablecida tasa de evapotranspiración.

\section{Resultados y discusión}

Propiedades químicas y físicas del suelo de los viñedos

Las propiedades físicas del suelo en ambas plantaciones (mayor y menor porosidad) muestran un $\mathrm{pH}$ moderadamente alcalino, un bajo contenido de sales (nitrógeno y fósforo), y contenido de carbonatos muy similar. El suelo del viñedo con menor porosidad tuvo $2.5 \%$ más potasio que el suelo con mayor porosidad (cuadro 1). Con relación a las propiedades físicas, se observó un contenido medio de materia orgánica del suelo en ambas superficies. El viñedo con mayor porosidad tuvo $25.82 \%$ más de contenido de poros y la densidad aparente fue $14.29 \%$ mayor, esto debido a un mayor contenido de arcilla (cuadro 2). La densidad de partículas fue muy similar en ambos viñedos.

\section{Contenido de agua en el suelo}

Las diferencias en la porosidad del suelo tuvieron un marcado efecto en el contenido de agua a través de las diferentes etapas de desarrollo del cultivo. Las variaciones fueron muy marcadas en el perfil de 0 a $30 \mathrm{~cm}$, mientras que en el de 30 a $60 \mathrm{~cm}$ no se notaron. Las plantas en el viñedo de mayor porosidad tuvieron mayor disponibilidad de agua durante todo el ciclo de crecimiento (figura 1), observándose también una menor variabilidad del contenido de humedad (promedios de media hora). Estudios previos han reportado que la capacidad de retención de agua del suelo está relacionada de manera directa con el contenido y tamaño de los poros (Tunc \& Sahin, 2015), y que la porosidad del suelo es función del contenido de arcilla (Szymański, Skiba, Wojtuń, \& Drewnik, 2015). En el cuadro 2 se muestra que la porosidad se asocia con un mayor contenido de arcilla y una menor densidad aparente del suelo.

Cuadro 1. Propiedades químicas del suelo de cada uno de los viñedos (con mayor y menor porosidad). Vinícola San Lorenzo,

Parras, Coahuila, México.

\begin{tabular}{l|c|c|c|c|c|c}
\hline \multicolumn{1}{c|}{ Suelo } & $\mathbf{p H}$ & $\begin{array}{c}\mathrm{CE} \\
\mathbf{( d s / m )}\end{array}$ & $\begin{array}{c}\text { Nitrógeno } \\
\mathbf{( \% )}\end{array}$ & $\begin{array}{c}\text { Fósforo } \\
\mathbf{( k g} / \mathbf{h a})\end{array}$ & $\begin{array}{c}\text { Potasio } \\
\mathbf{( k g} / \mathbf{h a})\end{array}$ & $\begin{array}{c}\text { Carbonatos } \\
\text { totales } \mathbf{( \% )}\end{array}$ \\
\hline $\begin{array}{l}\text { Mayor } \\
\text { porosidad }\end{array}$ & 8.57 & 1.01 & 0.10 & 67.32 & 524.7 & 43.67 \\
\hline $\begin{array}{l}\text { Menor } \\
\text { porosidad }\end{array}$ & 8.28 & 0.98 & 0.10 & 60.21 & 537.30 & 41.0 \\
\hline
\end{tabular}

Cuadro 2. Propiedades físicas del suelo de cada uno de los viñedos (con mayor y menor porosidad). Vinícola San Lorenzo, Parras, Coahuila, México.

\begin{tabular}{c|c|c|c|c|c|c|c|c}
\hline Suelo & $\begin{array}{c}\text { MO } \\
(\%)\end{array}$ & $\begin{array}{c}\text { Arcilla } \\
(\%)\end{array}$ & $\begin{array}{c}\text { Limo } \\
(\%)\end{array}$ & $\begin{array}{c}\text { Arena } \\
(\%)\end{array}$ & Textura & $\begin{array}{c}\text { Densidad } \\
\text { aparente } \\
\left(\mathbf{g} / \mathbf{c m}^{3}\right)\end{array}$ & $\begin{array}{c}\text { Densidad } \\
\text { de } \\
\text { partículas } \\
\left(\mathbf{g} / \mathbf{c m}^{3}\right)\end{array}$ & $\begin{array}{c}\text { Porosidad } \\
(\%)\end{array}$ \\
\hline $\begin{array}{c}\text { Mayor } \\
\text { porosidad }\end{array}$ & 1.98 & 50.0 & 26 & 24 & Arcilla & 1.26 & 2.048 & 0.385 \\
\hline $\begin{array}{c}\text { Menor } \\
\text { porosidad }\end{array}$ & 2.01 & 46.40 & 31.20 & 22.40 & $\begin{array}{c}\text { Migajón- } \\
\text { arcilloso }\end{array}$ & 1.44 & 2.075 & 0.306 \\
\hline
\end{tabular}



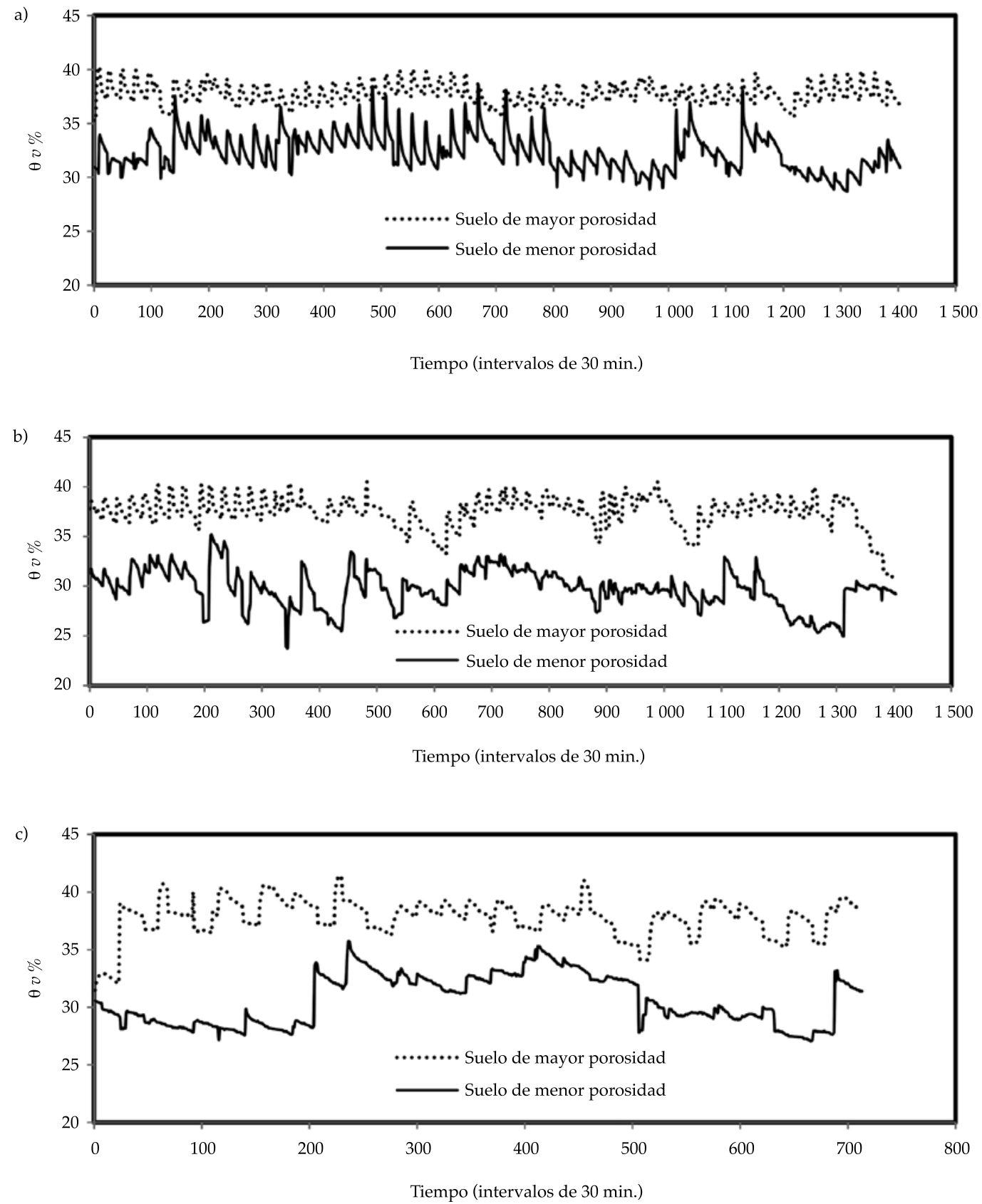

Figura 1. Cambios en la humedad del suelo en el perfil de 0 a $30 \mathrm{~cm}$ (promedios de $30 \mathrm{~min}$ ) durante el ciclo de producción del viñedo (mayo-agosto de 2014): a) desarrollo vegetativo; b) floración, y c) fructificación, en dos viñedos (cv Shiraz) en suelo de diferente porosidad. Vinícola San Lorenzo, Parras, Coahuila, México.

El contenido volumétrico promedio de agua en el suelo durante el ciclo de producción (mayo-agosto) fue mayor (Wilcoxon $\alpha \leq 0.05$ ) en la plantación de mayor porosidad (cuadro 3). En promedio, el contenido de agua en el suelo en el viñedo de mayor porosidad fue de $37.48 \%$ y en el de menor porosidad de $29.73 \%$, lo que representó una diferencia de $26.07 \%$. Una mayor disponibilidad de agua en el suelo para las plantas puede resultar en una tasa de transpiración 
Cuadro 3. Valores promedio (segmentos de $30 \mathrm{~min}$ ) de contenido de humedad volumétrico del suelo a través del ciclo de producción (mayo-agosto de 2014), de dos viñedos (cv Shiraz) con suelo de diferente porosidad. Vinícola San Lorenzo, Parras, Coahuila, México.

\begin{tabular}{c|c|c}
\hline Mes & $\begin{array}{c}\text { Suelo de mayor porosidad } \\
\Theta \mathbf{v} \%\end{array}$ & $\begin{array}{c}\text { Suelo de menor porosidad } \\
\Theta \mathbf{v} \%\end{array}$ \\
\hline Mayo & $37.8^{\mathrm{a}}$ & $30.7^{\mathrm{b}}$ \\
\hline Junio & $37.3^{\mathrm{a}}$ & $30.1^{\mathrm{b}}$ \\
\hline Julio & $37.2^{\mathrm{a}}$ & $29.1^{\mathrm{b}}$ \\
\hline Agosto & $37.6^{\mathrm{a}}$ & $29.0^{\mathrm{b}}$ \\
\hline
\end{tabular}

Nota: medias con diferente letra $\left({ }^{a}{ }^{b}\right)$ entre las columnas son estadísticamente diferentes (Wilcoxon 0.05).

y asimilación de bióxido de carbono más alta (Tagesson et al., 2015). Estudios hechos en un cultivo de maíz y girasol por Béziat et al. (2013) reportaron tasas diarias de ET de hasta $5.1 \mathrm{y}$ $5.6 \mathrm{~mm}$, respectivamente, debido a una mayor capacidad de retención de agua del suelo por un alto contenido de arcilla. Para un bosque de P. contorta, Mitchell, Emanuel y McGlynn (2015) reportaron una tasa baja de $E T(1.27 \mathrm{~mm})$ para condiciones de sequía, y que dicha tasa aumenta en forma proporcional con el contenido de agua en el suelo. En un cultivo de vid (Vitis vinifera L.) en clima seco, la tasa de $E T$ fue de $3.7 \mathrm{~mm}$ $\mathrm{d}^{-1}$ en periodos de baja humedad y de hasta 4.8 $\mathrm{mm} \mathrm{d}^{-1}$ para alto contenido de agua en el suelo (Atroosh, Mukred, \& Moustafa, 2013). También, Zhou, Zhou y Jia (2009) observaron que en un cultivo de carrizo (Phragmites australis), la humedad del suelo determinó la máxima tasa de fotosíntesis y de asimilación de bióxido de carbono. Para un bosque de eucalipto (Eucalyptus delegatensis), Keith, van Gorsel, Jacobsen y Cleugh (2012) encontraron que bajos contenidos de humedad del suelo del ecosistema afectan la tasa de fotosíntesis, así como en la vid; Flexas et al. (2010) observaron que la fotosíntesis es afectada por el cierre estomático debido a una baja humedad del suelo y que por lo común ocurre en las regiones vinícolas semiáridas.

Una tasa mayor de evapotranspiración y asimilación de bióxido de carbono, por una mayor disponibilidad de agua en el suelo, pueden afectar la eficiencia intrínseca del uso del agua del viñedo y la productividad marginal del agua.

\section{Eficiencia intrínseca del uso del agua}

La eficiencia intrínseca del uso del agua $\left(E F_{i}\right)$ de un ecosistema vegetal se puede definir como la relación entre la tasa de asimilación neta de bióxido de carbono (NEE) y la tasa de evapotranspiración (ET) ocurriendo de forma simultánea (Zermeño-Gonzalez et al., 2012; Ago et al., 2014). En la figura 2 se muestra que esta relación corresponde a una parábola decreciente, cuando el $N E E$ se grafica con valores negativos (indicando que el flujo de $\mathrm{CO}_{2}$ es hacia el dosel). Estudios previos ya han mostrado tal relación (Li et al., 2015; Yan et al., 2015), dicha tendenecia también implica que el NEE se incrementa (valores más negativos) a medida que la $E T$ aumenta. Esto se debe a que a mayor apertura de los estomas, el flujo de vapor de agua desde las hojas hacia la atmósfera y el de bióxido de carbono de la atmósfera hacia las hojas aumenta (Gowik \& Westhoff, 2011; Evans, 2013). Nótese que en el viñedo con mayor disponibilidad de agua en el suelo (por mayor porosidad), la asimilacion neta de bióxido de carbono (NEE) fue mayor (valores más negativos), de igual forma que la tasa de evapotranspiración (escala mayor del eje $\mathrm{x}$ de las gráficas de la figura 2). Estudios previos también han reportado incrementos de la tasa de ET y de NEE con mayor contenido de agua en el suelo. Por ejemplo, Yan et al. (2015), en un pastizal, encontraron que el NEE alcanza su tasa máxima cuando el ecosistema presenta valores altos de humedad en el suelo; mientras que Rutledge et al. (2014) observaron 
que durante la primavera (suelo con mayor humedad), la asimilación de bióxido de carbono del pasto Lolium perenne fue de 40 a 100\% mayor que durante los periodos de sequía. Se puede advertir también que el flujo de vapor de agua del dosel a la atmósfera es de una escala de mil mayor que el flujo de bióxido de carbono de la atmósfera al dosel; esto se debe al mayor peso molecular del $\mathrm{CO}_{2^{\prime}}$ y que la resistencia interna de las hojas al flujo de $\mathrm{CO}_{2}$ es mayor que la resistencia a la salida de vapor de agua (Jones, 1992; Nobel, 2009). Esto resulta en valores muy pequeños de la eficiencia intrínseca del uso del agua por los ecosistemas vegetales (Flexas et al., 2010; Gomes-Soares, Pereira-Tognella, Cuevas, \& Medina, 2015).

La tasa mayor de NEE y ET se observó en las etapas vegetativa y de floración (fases de mayor desarrollo foliar) en ambos viñedos (figura 2). Otros estudios en diferentes tipos de vegetación y en otros viñedos también han reportado tasas mayores de flujos de vapor de agua y bióxido de carbono en las etapas vegetativas y de floración (Li et al., 2015; Zhang et al., 2014).

Debido a que la relación entre NEE y la tasa de $E T$ es no lineal para evaluar la $E F_{i^{\prime}}$ que es la relación entre ambas (NEE/ET), es necesario definir una tasa de ET. Para los resultados de este estudio (figura 2) y para analizar la $E F_{i}$ en las diferentes etapas de desarrollo, en cada viñedo (de mayor y menor porosidad), se es-

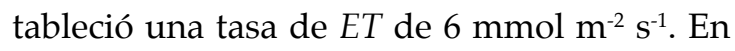
los valores del cuadro 4 se muestra que la $E F_{i}$ fue muy similar en la etapa vegetativa y de floración en ambos viñedos, y que en la etapa de fructificación se tuvo una notable reducción, más notable en el viñedo con mayor porosidad (cuadro 4). Esto probablemente ocurrió porque las hojas jóvenes en las etapas tempranas tienen mayor capacidad de asimilación de bióxido de carbono por un mayor contenido de pigmentos de clorofila (Yamori \& von Caemmerer, 2009; Raines, 2011), y que las hojas adultas tuvieron menor fijación de $\mathrm{CO}_{2}$ por una reducción del contenido de pigmentos de clorofila (Shimoda, Murayama, Mo, \& Oikawa, 2009; Zhou et al., 2009; Keith et al., 2012). La mayor disponibilidad de agua en el suelo por una mayor porosidad no se reflejó en una $E F_{i}$ más alta debido a que al aumentar la tasa de asimilación de $\mathrm{CO}_{2}$ también se incrementa la tasa de transpiración (Shimoda et al., 2009; Strilesky \& Humphreys, 2012).

La productividad marginal del agua (PMA) de los viñedos a través de las etapas de desarrollo de las plantas se evaluó derivando las funciones de $N E E$ contra la $E T$, que se muestran en la figura 2. En la figura 3 se observa que para una tasa de ET mayor de $4 \mathrm{mmol} \mathrm{m}^{-2} \mathrm{~s}^{-1}$, la $P M A$ fue mayor (valores más negativos) en el viñedo de mayor disponibilidad de agua en el suelo (por mayor porosidad) en las tres etapas de desarrollo. Por ejemplo, para la misma tasa de $E T$ de $6 \mathrm{mmol} \mathrm{m}^{-2} \mathrm{~s}^{-1}$ que se usó para determinar la eficiencia intrínseca del uso del agua, la PMA fue mayor en las plantas del viñedo con mayor contenido de agua en el suelo (mayor porosidad) en las tres etapas de desarrollo (figura 3). La PMA fue mayor en la etapa vegetativa (hojas más jóvenes) en ambos viñedos; en el de mayor porosidad fue $-1.408 \mu \mathrm{mol} \mathrm{CO} \mathrm{mmol}^{-1} \mathrm{H}_{2} \mathrm{O}$ y -1.126 $\mu \mathrm{mol} \mathrm{CO}_{2} \mathrm{mmol}^{-1} \mathrm{H}_{2} \mathrm{O}$ en el de menor porosidad, que correspondió a una diferencia de $31.44 \%$. En promedio, para las tres etapas de desarrollo, la PMA del agua de las plantas del viñedo con mayor porosidad (contenido más alto de agua en el suelo) fue $24.64 \%$ mayor. Estudios desarrollados por Rogiers y Clarke (2013) también identificaron que en un cultivo de vid cv Shiraz, la eficiencia del uso del agua en la etapa vegetativa fue mayor bajo condiciones de más contenido de agua en el suelo. De igual forma, Pagay (2016) menciona que en un estudio en vid cv Tempranillo, donde evaluaron riego deficitario, la eficiencia del uso del agua fue mayor bajo condiciones de riego óptimo, que la observada en condiciones de riego limitado.

Sin embargo, para valores bajos de la tasa de ET (menor de $4 \mathrm{mmol} \mathrm{m}^{-2} \mathrm{~s}^{-1}$ ), la PMA fue mayor en el viñedo de menor disponibilidad de agua en el suelo, esto se debe a que el cierre parcial de los estomas por una menor disponibilidad de agua en el suelo tiene un mayor impacto en la reducción del flujo de vapor de agua desde el interior de las hojas hacia la atmósfera que 

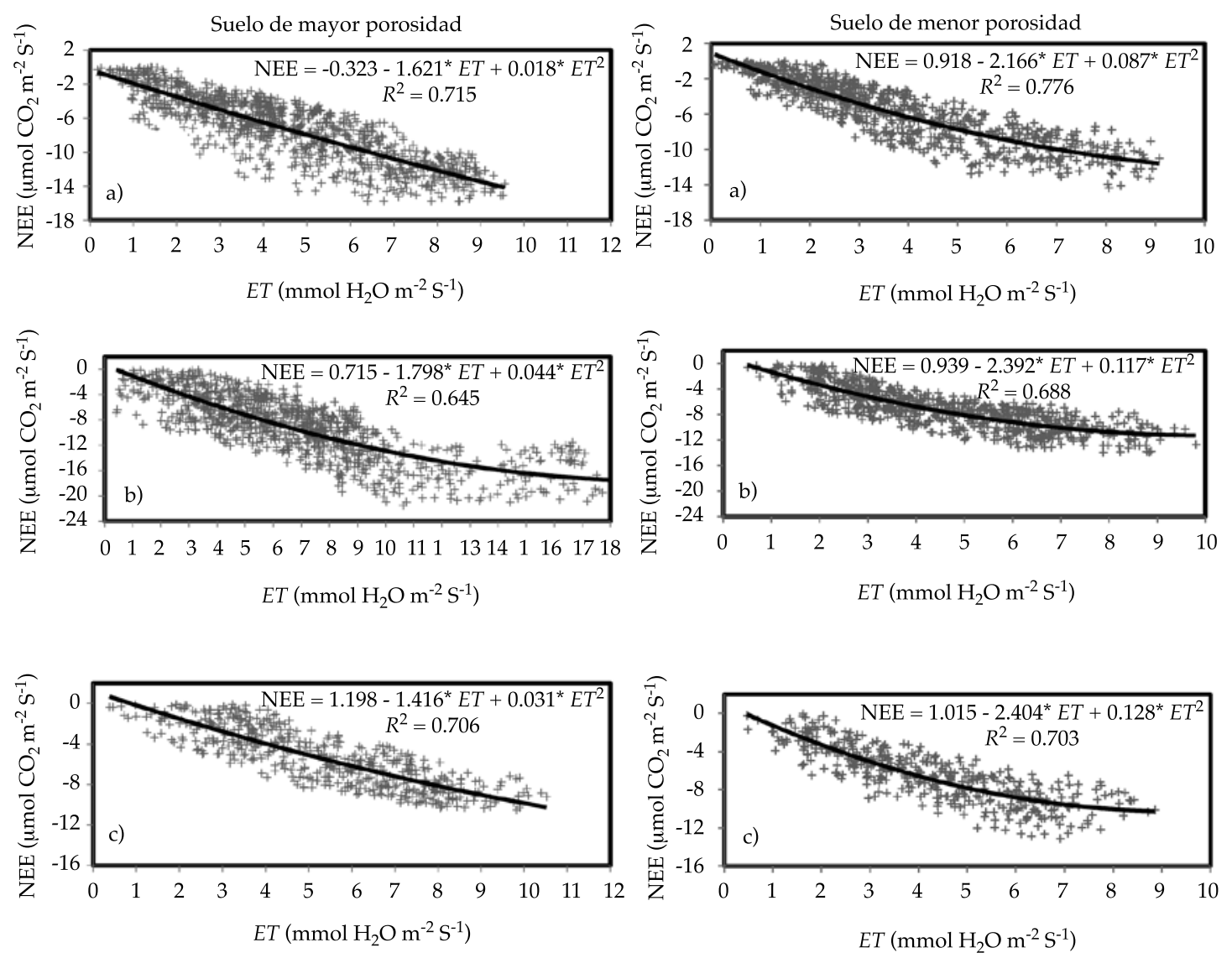

Figura 2. Relación entre la tasa de intercambio neto de bióxido de carbono (NEE) y la tasa de evapotranspiración (ET) a través de la etapa vegetativa (a), de floración (b) y de fructificación (c) en dos viñedos (cv Shiraz) de diferente porosidad del suelo. Vinícola San Lorenzo, Parras de la fuente, Coahuila, México, año de producción: 2014.

Cuadro 4. Eficiencia intrínseca del uso del agua a una tasa de evapotranspiración de $6 \mathrm{mmol} \mathrm{m}^{-2} \mathrm{~s}^{-1}$ en tres etapas de desarrollo de las plantas en dos viñedos de diferente porosidad. Vinícola, San Lorenzo, Parras, Coahuila, México, año de producción: 2014.

\begin{tabular}{|c|c|c|}
\hline Etapa de desarrollo & $\begin{array}{c}\text { Mayor porosidad } \\
\left(\mu \mathrm{mol} \mathrm{CO} / / \mathrm{mmol} \mathrm{H}_{2} \mathrm{O}\right)\end{array}$ & $\begin{array}{c}\text { Menor porosidad } \\
\left(\mu \mathrm{mol} \mathrm{CO} / / \mathrm{mmol} \mathrm{H}_{2} \mathrm{O}\right)\end{array}$ \\
\hline Vegetativa & -1.036 & -0.948 \\
\hline Floración & -0.961 & -1.009 \\
\hline Fructificación & -0.693 & -0.967 \\
\hline
\end{tabular}

en la circulación del bióxido de carbono de la atmósfera hacia el interior de las hojas debido a una mayor resistencia estomática al flujo del $\mathrm{CO}_{2}$ que al flujo del vapor de agua (Jones, 1992; Nobel, 2009), resultando en un valor mayor de la PMA.

\section{Conclusiones}

El viñedo con mayor porosidad tuvo una mayor disponibilidad de agua en el suelo durante el ciclo de crecimiento, que resultó en una mayor tasa de evapotranspiración y de 

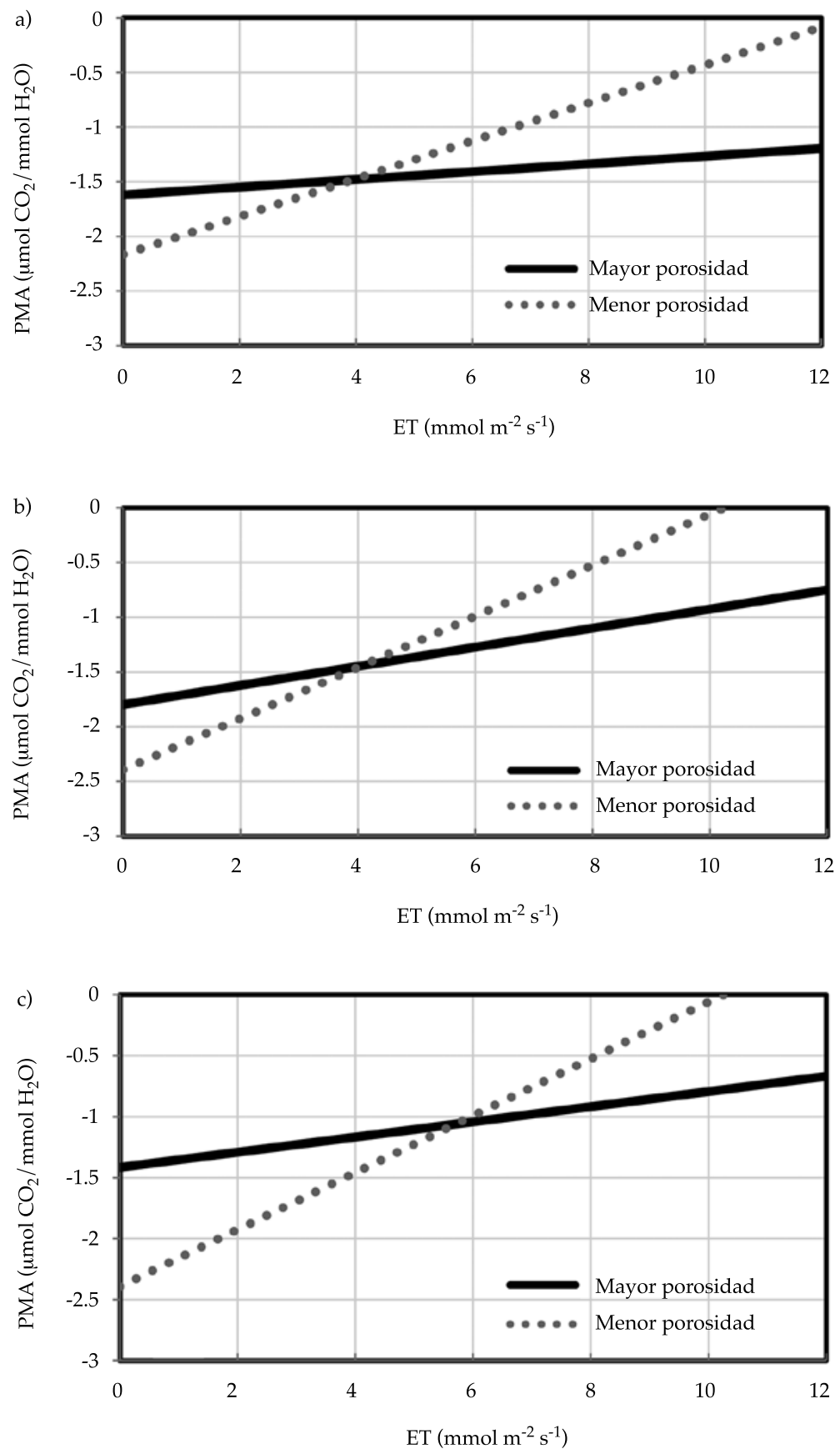

Figura 3. Productividad marginal del agua $(P M A)$ en función de la tasa de evapotranspiración a través de la etapa vegetativa (a), de floración (b) y de fructificación (c) en dos viñedos con diferente porosidad del suelo. Vinícola San Lorenzo, año de producción: 2014.

asimilación de bióxido de carbono, pero que no tuvo efecto en la eficiencia intrínseca del uso del agua $\left(E F_{i}\right)$. Sin embargo, la productividad marginal del agua para la misma tasa de evapotranspiración a la que se determinó la $E F_{i}$ fue mayor en el viñedo con mayor porosidad a través de las diferentes etapas de desarrollo de las plantas. 


\section{Referencias}

Ago, E. E., Agbossou, E. K., Galle, S., Cohard, J. M., Heinesch, B., \& Aubinet, M. (2014). Long term observations of carbon dioxide exchange over cultivated savanna under a Sudanian climate in Benin (West Africa). Agric. For. Meteorol., 197, 13-25.

Atroosh, K. B., Mukred, A. W. O., \& Moustafa, A. T. (2013). Water requirement of grape (Vitis vinifera) in the Northern Highlands of Yemen. J. Agric. Sci., 5(4), 136-145.

Béziat, P., Rivalland, V., Tallec, T., Jarosz, N., Boulet, G., Gentine, P., \& Ceschia, E. (2013). Evaluation of a simple approach for crop evapotranspiration partitioning and analysis of the water budget distribution for several crop species. Agric. For. Meteorol., 177, 46-56.

Bruschi-Gonçalves, R. A., Gloaguen, T. V., Folegatti, M. V., Libardi, P. L., Lucas, Y., \& Montes, C. R. (2010). Pore size distribution in soils irrigated with sodic water and wastewater. Revista Brasileira de Ciência Do Solo, 34(3), 701707.

El Economista (2013). El vino en México. El Economista. Fecha de consulta 16 de octubre del 2015. Recuperado de http: / / eleconomista.com.mx/entretenimiento/2013/06/03/ vino-mexico.

Evans, J. R. (2013). Improving photosynthesis. Plant Physiology, 162(4), 1780-1793.

Flexas, J., Galmés, J., Gallé, A., Gulías, J., Pou, A., RibasCarbo, M., Tomas, M., \& Medrano, H. (2010). Improving water use efficiency in grapevines: Potential physiological targets for biotechnological improvement. Australian Journal of Grape and Wine Research, 16, 106-121.

Gomes-Soares, M. L., Pereira-Tognella, M. M., Cuevas, E., \& Medina, E. (2015). Photosynthetic capacity and intrinsic water-use efficiency of rhizophora mangle at its southernmost western Atlantic range. Photosynthetica, 53(3), 464-470.

Gowik, U., \& Westhoff, P. (2011). The path from C3 to C4 photosynthesis. Plant Physiology, 155(1), 56-63.

Ham, J. M., \& Heilman J. L. (2003). Experimental test of density and energy-balance corrections on carbon dioxide flux as measured using open-path eddy covariance. Agron. J., 95(6), 1393-1403.

Holland, J. E., \& Biswas, A. (2015). Predicting the mobile water content of vineyard soils in New South Wales, Australia. Agric. Water Manag., 148, 34-42.

Hongshuo W., Rogers, J. C., \& Munroe, D. K. (2015). Commonly used drought indices as indicators of soil moisture in China. J. Hydrometeor., 16, 1397-1408.

INIFAP (2015). Fecha de consulta 03 de septiembre de 2015. Recuperado de http://clima.inifap.gob.mx/redinifap/ est.aspx?est=26796.

Jones, H. G. (1992). Plants and microclimate (428 pp.) (2nd. ed.). Cambridge, UK: Cambridge University Press.
Keith, H., van Gorsel, E., Jacobsen, K. L., \& Cleugh, H. a. (2012). Dynamics of carbon exchange in a Eucalyptus forest in response to interacting disturbance factors. Agric. For. Meteorol., 153, 67-81.

Kim, J., \& Verma, S. B. (1990). Components of the surface energy balance in a temperate grassland ecosystem. Boundary-Layer Meteorol., 51(4), 401-417.

Kustas, W. P., Prueger, J. H., Hatfield, J. L., Ramalingam, K., \& Hipps, L. E. (2000). Variability in soil heat flux from a mesquite dune site. Agric. For. Meteorol., 103(3), 249-264.

Lawson, T., \& Blatt, M. R. (2014). Stomatal size, speed, and responsiveness impact on photosynthesis and water use efficiency. Plant Physiol., 164(4), 1556-1570.

Li, S., Kang, S., Zhang, L., Du, T., Tong, L., Ding, R., \& Xiao, H. (2015). Ecosystem water use efficiency for a sparse vineyard in arid northwest China. Agric. Water Manag., 148, 24-33.

Lipiec, J., Walczak, R., Witkowska-Walczak, B., Nosalewicz, A., Słowińska-Jurkiewicz, A., \& Sławiński, C. (2007). The effect of aggregate size on water retention and pore structure of two silt loam soils of different genesis. Soil Tillage Res., 97(2), 239-246.

Liu, B., Cheng, L., Ma, F., Zou, Y., \& Liang, D. (2012). Growth, biomass allocation, and water use efficiency of 31 apple cultivars grown under two water regimes. Agrofor. Syst., 84(2), 117-129.

Mahrt, L., \& Vickers, D. (2002). Relationship of area-averaged carbon dioxide and water vapour fluxes to atmospheric variables. Agric. For. Meteorol., 112, 195-202.

Martens, C. S., Shay, T. J., Mendlovitz, H. P., Matross, D. M., Saleska, S. R., Wofsy, S. C., Woodward, W. S., Menton, M. C., De Moura, J. M. S., Crill, P. M., De Moraes, O. L. L., \& Lima, R. L. (2004). Radon fluxes in tropical forest ecosystems of Brazilian Amazonia: Night-time $\mathrm{CO}_{2}$ net ecosystem exchange derived from radon and eddy covariance methods. Glob. Chang. Biol., 10(5), 618-629.

Martínez-García, G., Pachepsky, Y., \& Vereecken, H. (2014). Effect of soil hydraulic properties on the relationship between the spatial mean and variability of soil moisture. J. Hydrol., 516, 154-160.

McAdam, S. M., \& Brodribb, T. J. (2014). Separating active and passive influences on stomatal control of transpiration. Plant Physiology, 164(4), 1578-1586.

Mitchell, S. R., Emanuel, R. E., \& McGlynn, B. L. (2015). Land-atmosphere carbon and water flux relationships to vapor pressure deficit, soil moisture, and stream flow. Agric. For. Meteorol., 208, 108-117.

Nobel, P. S. (2009). Physicochemical and environmental plant physiology. Boston: Academic Press.

OIV (2015). World Vitiviniculture Situation. Organización Internacional de la Viña y el Vino. Maniz, Alemania, julio de 2015.

Pagay, V. (2016). Effects of irrigation regime on canopy water use and dry matter production of Tempranillo' 
grapevines in the semi-arid climate of Southern Oregon, USA. Agricultural Water Management, 178, 271-280.

Pardo, N., Sánchez, M. L., Pérez, I. A., \& García, M. A. (2015). Energy balance and partitioning over a rotating rapeseed crop. Agric. Water Manag., 161, 31-40.

Poltoradnev, M., Ingwersen, J., \& Streck, T. (2014). Calibration and application of aquaflex TDT Soil water probes to measure the soil water dynamics of agricultural topsoil in southwest Germany. Irrig. Drain Eng., 141(6), 401-407.

Raines, C. A. (2011). Increasing photosynthetic carbon assimilation in C3 plants to improve crop yield: Current and future strategies. Plant Physiology, 155(1), 36-42.

Rogiers, S. Y., \& Clarke, S. J. (2013). Nocturnal and daytime stomatal conductance respond to root-zone temperature in 'Shiraz' grapevines. Annals of Botany, 111, 433-444.

Rutledge, S., Mudge, P. L., Wallace, D. F., Campbell, D. I., Woodward, S. L., Wall, A. M., \& Schipper, L. A. (2014). $\mathrm{CO}_{2}$ emissions following cultivation of a temperate permanent pasture. Agric. Ecosyst. Environ., 184, 21-33.

Scanlon, M. T., \& Albertson, J. D. (2004). Canopy scale measurements of $\mathrm{CO}_{2}$ and water vapor exchange along a precipitation gradient in southern Africa. Glob. Chang. Biol., 10, 329-341.

Shimoda, S., Murayama, S., Mo, W., \& Oikawa, T. (2009). Seasonal contribution of $\mathrm{C} 3$ and $\mathrm{C} 4$ species to ecosystem respiration and photosynthesis estimated from isotopic measurements of atmospheric $\mathrm{CO}_{2}$ at a grassland in Japan. Agric. For. Meteorol., 149(3-4), 603-613.

SIAP (2015). Servicio de información agroalimentaria y pesquera. Fecha de consulta: 16 de octubre de 2015. Recuperado de http://www.siap.gob.mx/ cierre-de-la-produccionagricola-por-cultivo/.

Song, L., Zhu, J., Yan, Q., Li, M., \& Yu, G. (2015). Comparison of intrinsic water use efficiency between different aged Pinus sylvestris var. Mongolic wide windbreaks in semiarid sandy land of northern China. Agrofor. Syst., 89(3), 477-489.

Strilesky, S. L., \& Humphreys, E. R. (2012). A comparison of the net ecosystem exchange of carbon dioxide and evapotranspiration for treed and open portions of a temperate peatland. Agric. For. Meteorol., 153, 45-53.

Sun, X. P., Yan, H. L., Kang, X. Y., \& Ma, F. W. (2013). Growth, gas exchange, and water-use efficiency response of two young apple cultivars to drought stress in two scion-one rootstock grafting system. Photosynthetica, 51(3), 404-410.

Szymański, W., Skiba, M., Wojtuń, B., \& Drewnik, M. (2015). Soil properties, micromorphology, and mineralogy of Cryosols from sorted and unsorted patterned grounds in the Hornsund area, SW Spitsbergen. Geoderma, 253, 1-11.

Tagesson, T., Fensholt, R., Cropley, F., Guiro, I., Horion, S., Ehammer, A., \& Ardö, J. (2015). Dynamics in carbon exchange fluxes for a grazed semi-arid savanna ecosystem in West Africa. Agric. Ecosyst. Environ., 205, 15-24.

Tunc, T., \& Sahin, U. (2015). The changes in the physical and hydraulic properties of a loamy soil under irrigation with simpler-reclaimed wastewaters. Agric. Water Manag., 158, 213-224.

Wang, Y., Hills, A., \& Blatt, M. R. (2014). Systems analysis of guard cell membrane transport for enhanced stomatal dynamics and water. Plant Phytologist, 164, 1593-1599.

Webb, E. K., Pearman, G. I., \& Leuning, R. (1980). Correction of flux measurements for density effects due to heat and water vapor transfer. Quart. J. Roy. Meteorol. Soc., 106(447), 85-100.

Xu, Y., Li, W., Shao, X., Xu, Z., \& Nugroho, P. (2014). Longterm trends in intrinsic water-use efficiency and growth of subtropical Pinus tabulaeformis carr. and Pinus taiwanensis Hayata in central China. Journal of Soils and Sediments, 14, 917-927.

Yamori, W., \& von Caemmerer, S. (2009). Effect of Rubisco activase deficiency on the temperature response of $\mathrm{CO}_{2}$ assimilation rate and Rubisco activation state: Insights from transgenic tobacco with reduced amounts of Rubisco activase. Plant Physiology, 151(4), 2073-2082.

Yan, W., Hu, Z., Zhao, Y., Zhang, X., Fan, Y., Shi, P., \& Li, Y. (2015). Modeling net ecosystem carbon exchange of alpine grasslands with a satellite-driven model. Plos One, 200, 119-128.

Yu, G., Song, X., Wang, Q., Liu, Y., Guan, D., Yan, J., \& Wen, X. (2007). Water-use efficiency of forest ecosystems in eastern China and its relations to climatic variables. New Phytologist, 177(4), 927-937.

Zermeño-González, A. (2007). Balance de energía sobre superficies vegetales y su aplicación en la agricultura. Memorias del XVII Congreso Nacional de Ingeniería Agrícola. Bermejillo, Durango, México, noviembre de 2007.

Zermeño-González, A., Villaltoro-Moreno, S., CortesBracho, J. J., Cadena-Zapata, M., Catalán-Valencia, E. A., García-Delgado, M. A., \& Munguía-López, J. P. (2012). Estimación del intercambio neto de $\mathrm{CO}_{2}$ en un cultivo de caña de azúcar durante el ciclo de plantilla. Agrociencia, 46, 579-591.

Zhang, Q., Wang, S., Li, L., Inoue, M., Xiang, J., Qiu, G., \& Jin, W. (2014). Effects of mulching and sub-surface irrigation on vine growth, berry sugar content and water use of grapevines. Agric. For. Meteorol., 143, 1-8.

Zhou, L., Zhou, G., \& Jia, Q. (2009). Annual cycle of $\mathrm{CO}_{2}$ exchange over a reed (Phragmites australis) wetland in Northeast China. Aquatic Botany, 91(2), 91-98.

\section{Dirección institucional de los autores}

M.C. Aaron Isain Melendres Alvarez

Dr. Alejandro Zermeño González

Universidad Autónoma Agraria Antonio Narro Departamento de Riego y Drenaje

Calzada Antonio Narro 1923

25315 Buenavista, Saltillo, Coahuila, México

Teléfonos: + 52 (844) 4110353 y 4110354

amelendres@uaaan.mx

azermeno@uaaan.mx 
Dr. Homero Ramírez Rodríguez

Universidad Autónoma Agraria Antonio Narro Departamento de Horticultura

Calzada Antonio Narro 1923

25315 Buenavista, Saltillo, Coahuila, MéxIco

Teléfono: + 52 (844) 4110353 y 4110354

homero.ramirez@uaaan.mx

M.C. José Omar Cárdenas Palomo

Palau Bioquim, S.A. de C.V.

Calle Salvador González Lobo 280, piso 2

Col. República Ote.

25280 Saltillo, Coahuila, México

Teléfono: + 52 (844) 416 4170, ext. 9

cardenasjomar@palaubioquim.com
Dr. Martín Cadena Zapata

Universidad Autónoma Agraria Antonio Narro Departamento de Maquinaria Agrícola Calzada Antonio Narro 1923

25315 Buenavista, Saltillo, Coahuila, Méxıco Teléfonos: + 52 (844) 4110353 y 4110354

martincadena@uaaan.mx 